\title{
Ion pair correlations due to interference between solvent polarizations induced in water
}

Cite as: J. Chem. Phys. 152, 064501 (2020); https://doi.org/10.1063/1.5133753

Submitted: 27 October 2019 . Accepted: 22 January 2020 . Published Online: 14 February 2020

Puja Banerjee, and Biman Bagchi (D)
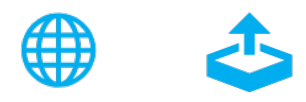

\section{ARTICLES YOU MAY BE INTERESTED IN}

\section{Machine learning for interatomic potential models}

The Journal of Chemical Physics 152, 050902 (2020); https://doi.org/10.1063/1.5126336

On the temperature dependence of liquid structure

The Journal of Chemical Physics 152, 011102 (2020); https://doi.org/10.1063/1.5135932

Studying rare events using forward-flux sampling: Recent breakthroughs and future outlook

The Journal of Chemical Physics 152, 060901 (2020); https://doi.org/10.1063/1.5127780

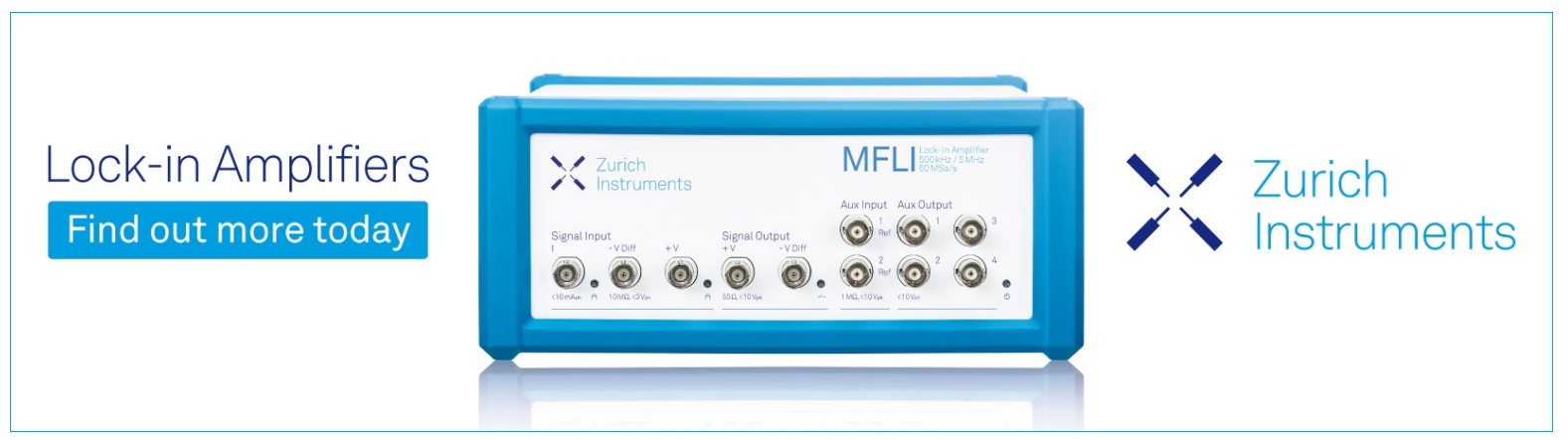




\title{
Ion pair correlations due to interference between solvent polarizations induced in water
}

\author{
Cite as: J. Chem. Phys. 152, 064501 (2020); doi: 10.1063/1.5133753 \\ Submitted: 27 October 2019 - Accepted: 22 January 2020 • \\ Published Online: 14 February 2020
}

Puja Banerjee and Biman Bagchi ${ }^{a}$

\section{AFFILIATIONS}

Solid State and Structural Chemistry Unit, Indian Institute of Science, Bangalore, Karnataka 560012, India

\author{
a) Author to whom correspondence should be addressed: profbiman@gmail.com
}

\begin{abstract}
Motions of two distinct ions can get correlated because the polarization induced by the ions can propagate through intervening water and can interfere with each other. This important aspect, which is not included in the continuum model based theories, has not been studied adequately. We calculate the effective force between two oppositely charged and similarly charged ions fixed in water as a function of separation distance R. At short separations, $R$ less than $1.5 \mathrm{~nm}$, the effective force vastly differs from the $1 / \varepsilon_{s} R^{2}$ dependence advocated by the screened Coulomb's force law (SCFL), where $\varepsilon_{s}$ is the static dielectric constant of the medium. This breakdown of the SCFL is shown to be due to the persistent interference between the polarizations created by the two charges in a manner similar to the vortex-antivortex pair formation in the XY model Hamiltonian. The distance dependence of dielectric constants, $\varepsilon_{s}(\mathrm{R})$, extracted from our simulation exhibits interesting features and can be used in future modeling. In addition, we show that the force-force time autocorrelation between two neighboring ions decays differently at short separation and analyze the friction on the ion pair at different separation distances.
\end{abstract}

Published under license by AIP Publishing. https://doi.org/10.1063/1.5133753

\section{INTRODUCTION}

The role of solvents in the structure and dynamics of electrolyte solutions has been a central research area of physical chemistry, being active for more than a century. ${ }^{1-10}$ In particular, interaction between two charges in a polar solvent plays an important role in many chemical and biological reactions. These studies inevitably use Coulomb's law (CL) to model electrostatic interactions among the charges. A notable example is found in Marcus's theory of electron transfer. In an aqueous solution of two ions at a separation $\mathrm{R}$, the solvent dipoles tend to orient themselves in such a way that the interaction can be screened, and when two different ions separated by a distance $\mathrm{R}$ act simultaneously on the same solvent molecule, it gives rise to an interference whose effects on interaction are hard to quantify.

In order to reduce the enormous complexity in dealing with the many-body systems with free or bound charges, the interaction between two charges immersed in a dipolar liquid is often modeled by a screened Coulomb's force law (SCFL), where the bare Coulomb's potential is divided (screened) by the static dielectric constant of the medium, $\varepsilon_{s}$. While the bare Coulomb's force law $(B C F L)$ is exact in vacuum, the SCFL is only approximate. According to Coulomb's law (CL), the expression of the bare force between two ions $\mathrm{A}$ and $\mathrm{B}$ having charges $\mathrm{q}_{\mathrm{A}}$ and $\mathrm{q}_{\mathrm{B}}$ separated by a distance $\mathrm{R}$ is given by

$$
F_{B C F L}\left(R, q_{A}, q_{B}\right)=\frac{q_{A} q_{B}}{R^{2}} .
$$

As mentioned above, it is usual, in the condensed phase, to replace this force by the screened form,

$$
F_{S C F L}\left(R, q_{A}, q_{B}\right)=\frac{q_{A} q_{B}}{\varepsilon_{S} R^{2}} .
$$

For similar charges, this force is repulsive, and for opposite charges, this is attractive, at all separations. 
An insightful derivation of the SCFL [Eq. (2)] can be obtained using the polarization field created by ions in the dipolar liquid. ${ }^{2,3,11,12}$ In this model, the polarization field, $\mathbf{P}(\mathbf{r})$, depends on the orientations of solvent molecules at a position $\mathbf{r}$. It produces an electrostatic potential $\mathrm{V}_{\mathrm{A}}$ at position $\mathrm{A}$, which is given by

$$
\mathrm{V}_{\mathrm{A}}=-\int_{\mathrm{r}>\mathrm{A}} \frac{\mathbf{P}(\mathbf{r}) \cdot \mathbf{r}}{|\mathbf{r}|^{3}} \mathrm{~d} \mathbf{r} .
$$

The free energy of a polarization field in a dielectric medium is given by $^{3,1}$

$$
\Pi=2 \pi\left(1-\frac{1}{\varepsilon_{\mathrm{s}}}\right)^{-1} \int_{\substack{\left|\mathbf{r}-\mathbf{r}_{\mathrm{A}}\right|>\mathrm{a} \\\left|\mathbf{r}-\mathbf{r}_{\mathrm{B}}\right|>\mathrm{b}}}[\mathbf{P}(\mathbf{r})]^{2} \mathrm{~d} \mathbf{r} .
$$

If we have two ions, $A$ and $B$ with charges $\mathrm{q}_{A}$ and $\mathrm{q}_{\mathrm{B}}$, the free energy of the system is given by

$$
\begin{aligned}
\Pi= & 2 \pi\left(1-\frac{1}{\varepsilon_{\mathrm{S}}}\right)^{-1} \int_{\substack{\left|\mathbf{r}-\mathbf{r}_{\mathrm{A}}\right|>\mathrm{a} \\
\left|\mathbf{r}-\mathbf{r}_{\mathrm{B}}\right|>\mathrm{b}}}[\mathbf{P}(\mathbf{r})]^{2} \mathrm{~d} \mathbf{r} \\
& -\int_{\substack{\left|\mathbf{r}-\mathbf{r}_{\mathrm{A}}\right|>\mathrm{a} \\
\left|\mathbf{r}-\mathbf{r}_{\mathrm{B}}\right|>\mathrm{b}}} \mathbf{P}(\mathbf{r})\left[\frac{\mathrm{q}_{\mathrm{A}}\left(\mathbf{r}-\mathbf{r}_{\mathrm{A}}\right)}{\left|\mathbf{r}-\mathbf{r}_{\mathrm{A}}\right|^{3}}-\frac{\mathrm{q}_{\mathrm{B}}\left(\mathbf{r}-\mathbf{r}_{\mathrm{B}}\right)}{\left|\mathbf{r}-\mathbf{r}_{\mathrm{B}}\right|^{3}}\right] \mathrm{d} \mathbf{r} .
\end{aligned}
$$

The equilibrium polarization, $\mathbf{P}(\mathbf{r})$, is obtained by minimizing the free energy expression with the constraint of the potential from the two charges. In the polarization field, $\mathbf{P}(\mathbf{r})$, the electrostatic potential difference between point $A$ and point $B$ is given by

$$
\Delta \mathrm{V}=-\int_{\substack{\left|\mathrm{r}-\mathrm{r}_{\mathrm{A}}\right|>\mathrm{a} \\\left|\mathrm{r}-\mathrm{r}_{\mathrm{B}}\right|>\mathrm{b}}} \mathbf{P}(\mathbf{r})\left[\frac{\mathbf{r}-\mathbf{r}_{\mathrm{A}}}{\left|\mathbf{r}-\mathbf{r}_{\mathrm{A}}\right|^{3}}-\frac{\mathbf{r}-\mathbf{r}_{\mathrm{B}}}{\left|\mathbf{r}-\mathbf{r}_{\mathrm{B}}\right|^{3}}\right] \mathrm{d} \mathbf{r},
$$

where $r_{A}$ and $r_{B}$ denote the positions of $A$ and $B$, respectively.

Now, the free energy of the system of two charges is a functional of $\mathbf{P}(\mathbf{r})$. For different electrostatic potentials between two charges $(\Delta \mathrm{V})$, the free energy and equilibrium polarization profiles, $\mathbf{P}(\mathbf{r})$, are different. According to Euler's variational principle, the polarization function, $\mathbf{P}(\mathbf{r})$, which minimizes the free energy $\Pi$ subject to the requirement of Eq. (6), can be determined by minimizing the functional,

$$
\begin{aligned}
\mathrm{F}= & 2 \pi\left(1-\frac{1}{\varepsilon_{\mathrm{S}}}\right)^{-1} \int_{\substack{\left|\mathbf{r}-\mathbf{r}_{\mathrm{A}}\right|>\mathrm{a} \\
\left|\mathbf{r}-\mathbf{r}_{\mathrm{B}}\right|>\mathrm{b}}}[\mathbf{P}(\mathbf{r})]^{2} \mathrm{~d} \mathbf{r} \\
& -\int_{\substack{\left|\mathbf{r}-\mathbf{r}_{\mathrm{A}}\right|>\mathrm{a} \\
\left|\mathbf{r}-\mathbf{r}_{\mathrm{B}}\right|>\mathrm{b}}} \mathbf{P}(\mathbf{r})\left[\frac{\mathrm{q}_{\mathrm{A}}\left(\mathbf{r}-\mathbf{r}_{\mathrm{A}}\right)}{\left|\mathbf{r}-\mathbf{r}_{\mathrm{A}}\right|^{3}}-\frac{\mathrm{q}_{\mathrm{B}}\left(\mathbf{r}-\mathbf{r}_{\mathrm{B}}\right)}{\left|\mathbf{r}-\mathbf{r}_{\mathrm{B}}\right|^{3}}\right] \mathrm{d} \mathbf{r} \\
& +\alpha\left[-\int_{\substack{\left|\mathbf{r}-\mathbf{r}_{\mathrm{A}}\right|>\mathrm{a} \\
\left|\mathbf{r}-\mathbf{r}_{\mathrm{B}}\right|>\mathrm{b}}} \mathbf{P}(\mathbf{r})\left[\frac{\mathbf{r}-\mathbf{r}_{\mathrm{A}}}{\left|\mathbf{r}-\mathbf{r}_{\mathrm{A}}\right|^{3}}-\frac{\mathbf{r}-\mathbf{r}_{\mathrm{B}}}{\left|\mathbf{r}-\mathbf{r}_{\mathrm{B}}\right|^{3}}\right] \mathrm{d} \mathbf{r}-\Delta \mathrm{V}\right],
\end{aligned}
$$

where $\alpha$ is a constant (Lagrange multiplier) to be determined. By minimizing this functional with respect to the variation of $\mathbf{P}(\mathbf{r})$, we obtain the expression of $\mathbf{P}(\mathbf{r})$ that can be used to derive an expression for polarization induced potential energy difference between two charged spheres A and B,

$$
\mathrm{E}_{\mathrm{s}}=\frac{1}{2}\left(1-\frac{1}{\varepsilon_{\mathrm{s}}}\right)\left[\frac{\mathrm{q}_{\mathrm{A}}^{2}}{\mathrm{a}}+\frac{\mathrm{q}_{\mathrm{B}}^{2}}{\mathrm{~b}}+\frac{2 \mathrm{q}_{\mathrm{A}} \mathrm{q}_{\mathrm{B}}}{\mathrm{R}}\right],
$$

where $\mathrm{q}_{\mathrm{A}}$ and $\mathrm{q}_{\mathrm{B}}$ are the charges on two spheres with radii $\mathrm{a}$ and $\mathrm{b}$, respectively, and $\mathrm{R}$ is the distance between centers of two spheres.

Now, the derivative of solvent reorganization energy $\left(\mathrm{E}_{\mathrm{s}}\right)$ gives the polarization induced force (PIF),

$$
\mathrm{F}_{\mathrm{PIF}}=-\left(1-\frac{1}{\varepsilon_{\mathrm{S}}}\right) \frac{\mathrm{q}_{\mathrm{A}} \mathrm{q}_{\mathrm{B}}}{\mathrm{R}^{2}}
$$

This PIF is attractive for two similar charges and repulsive for opposite charges. By adding this screening force to the force from bare Coulomb's force law ( $\mathrm{F}_{\mathrm{BCFL}}$ ), we get the screened Coulomb's force with $\varepsilon_{s}$ in the denominator. We note in passing that the present discussion parallels that in the celebrated Marcus theory of electron transfer, ${ }^{1,2,13}$ where an expression for solvent reorganization energy $\left(E_{s}\right)$ between two charged spheres is derived by using the similar approach. Since this derivation is based entirely on the continuum framework and no respect is given to the molecular nature of the liquid, its validity is questionable, especially at small-to-intermediate values of separation $R$.

A long time ago, Debye ${ }^{3}$ pointed out that the SCFL can breakdown close to an ion because the dielectric constant can become position dependent and should be replaced by $\varepsilon_{s}(r)$, as often practiced in theories and simulations, particularly for biomolecules. The most simple approach is to use $\varepsilon_{s}(r)$ as a linear function of distance from the ion/charge $(r),{ }^{14,15}$ that is, $\varepsilon_{s}(r)=C r$, where $\mathrm{C}$ is a constant generally assigned with the values ranging from 1 to 4.5 . In an influential study using the continuum model, Warshel et al. developed a more accurate functional form of $\varepsilon_{s}(r)$ that varies exponentially with distance. ${ }^{16}$ Despite a large number of theoretical studies focused on the construction of a model dielectric function in different biological systems (Srinivasan and Olson, ${ }^{17}$ Hingerty and co-workers, ${ }^{18}$ and Ramstein and Lavery ${ }^{19}$ ), the rational for such replacement is often not clear, but nevertheless it serves a practical purpose in simulations. However, all these continuum model descriptions neglect the molecular nature of the solvent. There is a different, not quite anticipated, consequence of this neglect of molecularity. Recently, Schaaf and Gekle ${ }^{20}$ calculated the local dielectric function, $\varepsilon(\mathrm{r})$, in the hydration layer around charged and uncharged spherical solutes from molecular dynamics (MD) simulations with explicit solvents and obtained interesting insights into the dielectric response of uncharged, positively charged, and negatively charged solutes.

Several theoretical ${ }^{21-23}$ and experimental ${ }^{24}$ studies have shown that due to the long-range nature of polar interaction, the polarization induced by any of the changes may persist up to a long distance. Long ago, Høye and Stell ${ }^{25,26}$ derived ion-ion, ion-solvent, and solvent-solvent pair distribution functions by including the molecularity of the solvent rather than structureless continuum treatment. This seminal study predicted a long-range correlation of solvent molecules in dilute salt solutions. In a recent experiment, Chen et al. ${ }^{27}$ demonstrated the existence of such a correlation in water at a large length scale (even beyond $8 \mathrm{~nm}$ ) in the 
presence of ions. To explain this experimental study, Pluhařová and co-workers ${ }^{28}$ carried out a simulation study that seems to show that the long-range order of the water-water orientational correlation function could indeed be influenced by ion-ion correlation as it affects their individual solvation shells.

The interference of polarization created by two ions that modify the force between them was not investigated. An example of such interference was discussed in Ref. 29 that considered water within reverse micelles. The interference can be destructive (for two positive ions) and constructive (for oppositely charged ions).

Earlier in a mode-coupling theory analysis of diffusion and viscosity, it was pointed out that the molecular nature of ionsolvent interaction can play an important role even at low concentrations. ${ }^{30-32}$ It was also suggested that local orientational correlations can be used to obtain a wavenumber dependent dielectric function, $\varepsilon(k)$, using classical density functional theory (DFT).

In the present work, we extract the distance dependent dielectric function, $\varepsilon_{s}(\mathrm{R})$, from a full atomistic simulation of an ion pair in water, where $\mathrm{R}$ is the separation distance of the ion pair.

Our simulations reveal that screened Coulomb's potential loses its validity at distances less than 1-1.5 $\mathrm{nm}$ in liquid water for monatomic ions. The force between two similar charges is found to be more repulsive and that of two opposite charges more attractive by a large factor even in the absence of any other ionic species in the system.

\section{SYSTEM AND SIMULATION DETAILS}

Molecular dynamics (MD) simulations of potassium chloride $(\mathrm{KCl})$ in water have been carried out using the Lammps package. ${ }^{34}$ Rigid non-polarizable force field parameters have been used for water as well as ions. The SPC/E model ${ }^{35}$ has been employed for water. For ions, potential parameters from Ref. 36 have been employed. The self-interaction parameters are listed in Table I and consist of Lennard-Jones and Coulombic terms.

We have taken two types of systems. In the first type, we have taken $1 \mathrm{~K}^{+}$and $1 \mathrm{Cl}^{-}$ions in a cubic simulation cell of length $70.0 \AA$ with 11319 water molecules, and the positions of the $\mathrm{K}^{+}$ and $\mathrm{Cl}^{-}$ions have been kept fixed with distances varying from 5 to $15 \AA$.

In the second type, we have taken two $\mathrm{K}^{+}$ions in the same simulation cell and the positions of the ions have been kept fixed with different distances between them. This is a system with no negative charges to see only the effect of water on the interaction of

TABLE I. Values of Lennard-Jones and electrostatic interaction potential parameters. e represents the magnitude of the electronic charge.

\begin{tabular}{llccc}
\hline \hline Atom, $i$ & $\sigma_{i i}(\AA)$ & $\varepsilon_{i i}(\mathrm{~kJ} / \mathrm{mol})$ & $q_{i}(e)$ & Reference \\
\hline $\mathrm{H}^{\mathrm{w}}$ & 0.000 & 0.000 & +0.4238 & 35 \\
$\mathrm{O}^{\mathrm{w}}$ & 3.169 & 0.6502 & -0.8476 & 35 \\
$\mathrm{~K}^{+}$ & 3.331 & 0.4184 & +1.0 & 36 \\
$\mathrm{Cl}^{-}$ & 4.40 & 0.4184 & -1.0 & 36 \\
\hline \hline
\end{tabular}

two $\mathrm{K}^{+}$ions. Note that one should be careful with such a simulation with a non-zero net charge. The use of grid based electrostatic calculations (Ewald summation) with the periodic boundary condition can result into infinite electrostatic energy by adding up interactions with periodic images with only positive charges. However, here we obtained good energy conservation because of the fact that two positive charges are kept fixed in a sufficiently large box.

Constrained MD simulations were carried out by fixing the positions of ions at a particular distance of separation in the canonical ensemble with periodic boundary conditions with a cut-off radius of $15 \AA$. The long-range forces were computed with Ewald summation. ${ }^{37,38}$ The trajectory was propagated using a velocity Verlet integrator with a time step of $1 \mathrm{fs}$. The aqueous $\mathrm{KCl}$ system was equilibrated for 300 ps at $298 \mathrm{~K}$, and then a $6 \mathrm{~ns}$ MD trajectory was generated in the canonical (NVT) ensemble. The coordinates were stored every $10 \mathrm{fs}$ for subsequent use for the evaluation of various properties. Finally, we reported the results averaged from several different trajectories.

We also have simulated only one $\mathrm{K}^{+}$ion and only one $\mathrm{Cl}^{-}$ion fixed in a particular position of the water box to compute the difference map of polarization shown in the supplementary material (Fig. S-1)

\section{RESULTS AND DISCUSSION}

\section{A. Polarization field created in water around an ion}

It is evident that the water molecules located near a given ionic species become oriented in a particular direction due to ion-dipole interaction. Polar solvents, other than water, such as methanol and dimethyl sulfoxide (DMSO), also exhibit such rearrangements of solvent dipoles around charged species. We defined a molecular dipole of water molecule and measured the angle between the dipole vector and the ion-water connecting vector $(\theta)$ [Fig. 1(a)]. Due to the nature of ion-water interaction, polarization measured by $\cos \theta$ possesses inverse profiles for the two systems of similarly charged ions and oppositely charged ions but is not actually a mirror image of each other. This has already been suggested in earlier studies that the water solvation shell around a positively charged ion is more structured than that around a negatively charged ion. The polarization profiles exhibit two major peaks, and having higher polarity, polarization of water extends slightly larger than $10 \AA$ [see Fig. 1(b)].

\section{B. Interference between the two induced polarizations in water}

We carried out a series of long atomistic simulations with ion pairs fixed in water with constrained molecular dynamics at different distances of separation between them. Our main aim was to analyze the polarization of water in the presence of two fixed charges and the modified force between two charges due to the polarization force. The resultant value of polarization for two oppositely charged ions along the inter-ionic axis is determined by the constructive interference of polarization [Fig. 2(a)]. Due to this, the polarization of water between two oppositely charged ions with a shorter inter-ionic distance increases, which in turn modifies the force acting between 

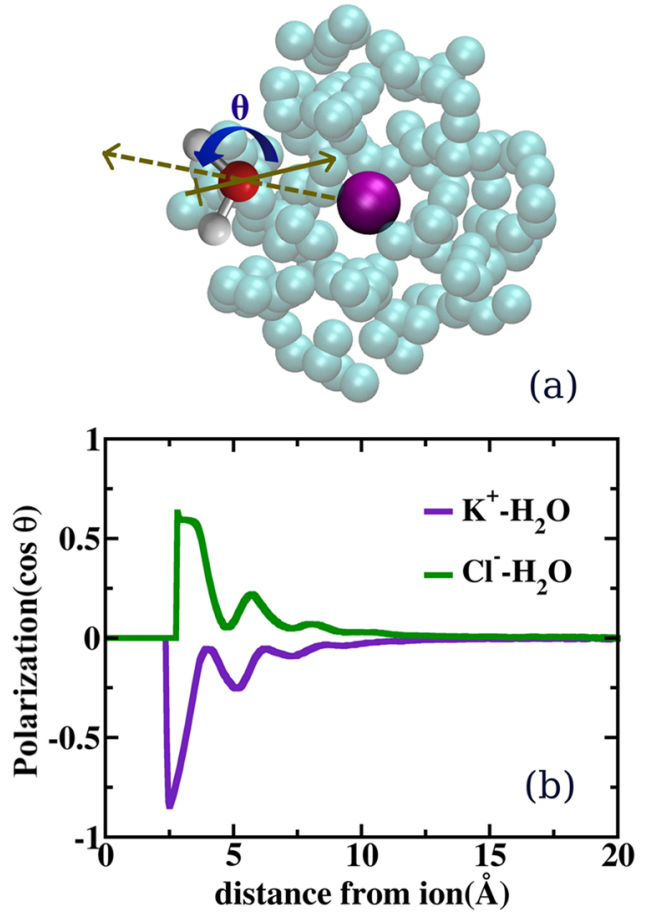

FIG. 1. (a) Hydration shell around an ion and representation of the polarization angle, $\theta$, between water dipole and ion-water intermolecular axis. (b) Polarization profiles of water around isolated positive and negatively charged ions.

two oppositely charged ions that we shall discuss later in detail. In Fig. 2(b), the average dipole vectors of water molecules is plotted around two ions, $\mathrm{K}^{+}$and $\mathrm{Cl}^{-}$. The situation is tantalizingly analogous to the formation of vortex-antivortex pair as found in the XY model Hamiltonian. It is evident that the water molecules located in between the two ions (near to the inter-ionic axis) are oriented in the same direction through out the inter-ionic region. Figure 2(c) shows the polarization profiles in the presence of two oppositely charged ions obtained from the simulation. Here, polarization has a structural pattern; hence, the unique nature of constructive interference at a different distance of separation finally dictates the attractive force between them. In the supplementary material (Fig. S-1), we have shown the difference map of polarization in this ion pair system compared to the single ion situations. This clearly shows the effect of constructive interference of two polarization profiles induced by two ions.

Next, we focus on two positively charged ion pairs. Here, polarization of water molecules between two ions along the inter-ionic axis experiences a destructive interference [Fig. 3(a)]. Figure 3(b) shows that the dipole vectors in the central region of inter-ionic axis do not have any particular reorientation due to a lower polarization of water molecules. This modifies the force between two positively charged ions such that at shorter separation, they experience much more repulsion than that predicted by screened Coulomb's law. Figure 3(c) shows the polarization profiles in the presence of two positively charged ions obtained from the simulation.
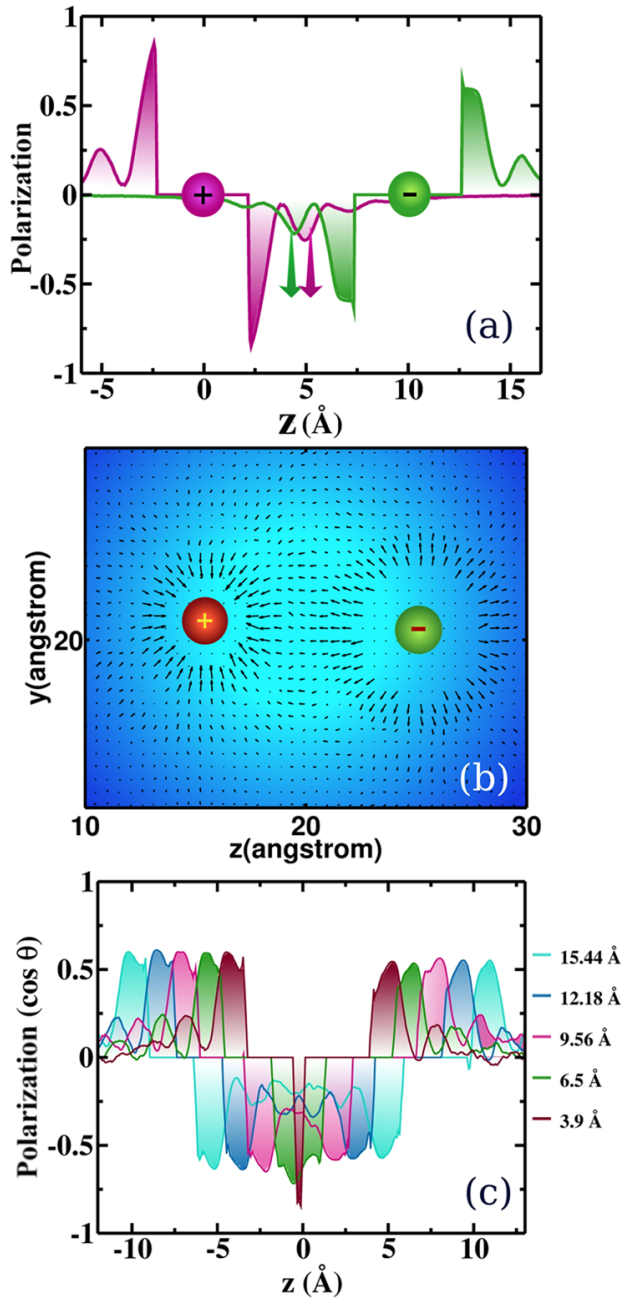

FIG. 2. (a) The polarization interaction of water in the presence of two oppositely charged ions at a separation of $\sim 10 \AA$. (b) Average direction of dipole vectors around that $\mathrm{K}^{+}$and $\mathrm{Cl}^{-}$ion pairs for the same ion pair separation distance. (c) Polarization profiles obtained from simulation along the inter-ionic axis of the ion pair for different separation distances.

\section{Effective force between two charges immersed in water}

We computed the polarization-induced force acting between the two ions and added this to the bare Coulomb's force to obtain the effective total force between two ions. Toward this, we first measure the total electrostatic force on ion n $_{1}$ exerted by all the water molecules $\left(F_{1}\right)$ and the total force on ion $_{2}$ exerted by all water molecules $\left(F_{2}\right)$. Then, we project $F_{1}$ and $F_{2}$ on the inter-ionic axis and determine the polarization induced force $\left(\mathrm{F}_{\mathrm{PIF}}\right)$ between the two ions using the projected forces $F_{1 P}$ and $F_{2 P}$, respectively, which is repulsive for two oppositely charged ions and attractive for two similar ions, therefore screening Coulomb's force between them. The effective force $\left(\mathrm{F}_{\text {eff }}\right)$ between two ions can be obtained by adding this force with 

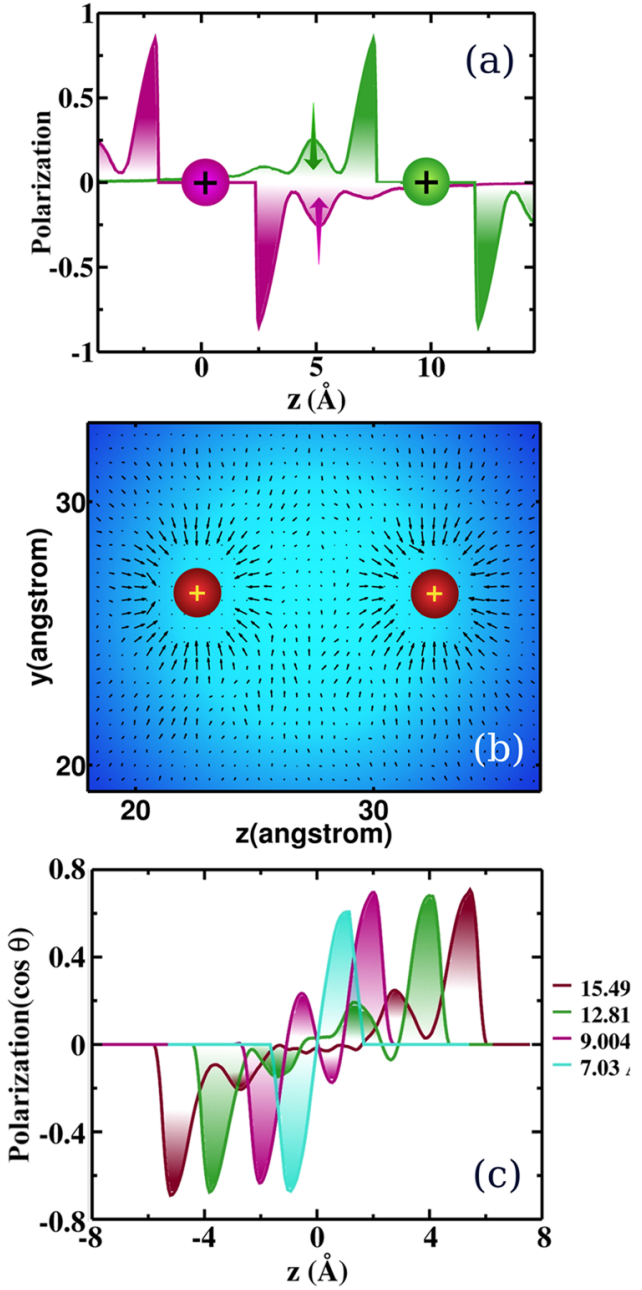

FIG. 3. (a) The polarization interaction of water in the presence of two positively charged ions at a separation of $\sim 10 \AA$. (b) Average direction of dipole vectors around the two $\mathrm{K}^{+}$ions for the same separation distance. (c) Polarization profiles obtained from simulation along the inter-ionic axis of the ion pair for different separation distances.

Coulomb's bare force $\left(\mathrm{F}_{\mathrm{BCFL}}\right)$. As $\mathrm{F}_{\mathrm{PIF}}$ and $\mathrm{F}_{\mathrm{BCFL}}$ are similar in magnitude (for water) but opposite in sign, the effective force is smaller by a factor of $\sim 50-100$, which makes it difficult to get the values accurately. To overcome this, we created several long trajectories with frequently saved data to generate sufficient data points to obtain an accurate average. A pair of $\mathrm{K}^{+}$and $\mathrm{Cl}^{-}$has been taken with positions fixed in water, the distance of separation is changed within a range of 5-15 $\AA$ in a series of simulations, and the different contributions to the effective force between them are shown in Fig. 4(a). Here, the polarization force is found to be repulsive, which screens the attractive Coulomb's force between $\mathrm{K}^{+}$and $\mathrm{Cl}^{-}$. Figure 4(b) shows the effective distance-dependent force for the system of two $\mathrm{K}^{+}$ions in water where the polarization induced force, $\mathrm{F}_{\mathrm{PIF}}$, is seen to be attractive here that screens Coulomb's repulsive force between two positive ions and gives the effective force between them.
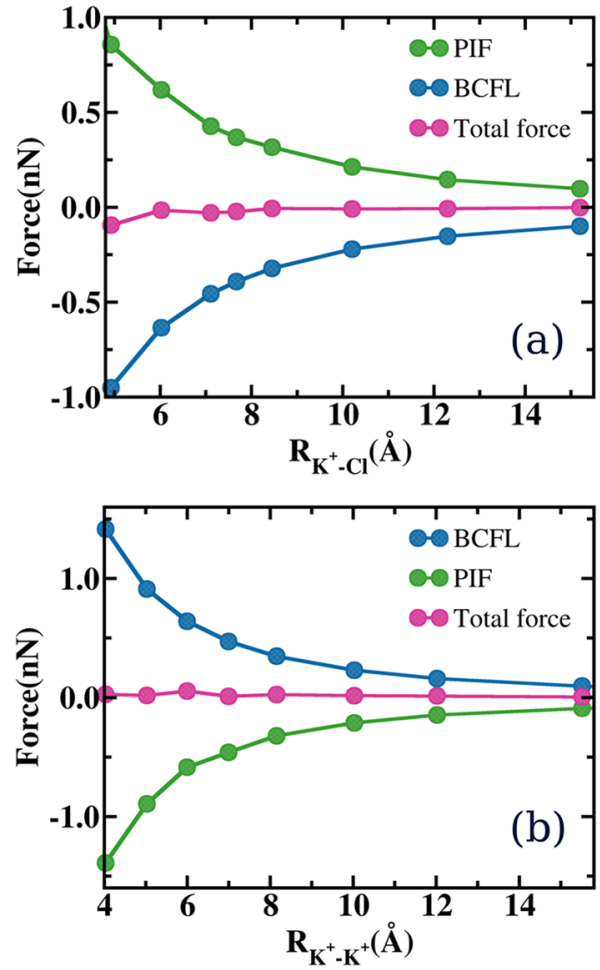

FIG. 4. (a) Distance dependence of forces between $\mathrm{K}^{+}$and $\mathrm{Cl}^{-}$ions in water from bare Coulomb's force law (BCFL), the polarization induced force (PIF) from simulation and the total force between them, (b) corresponds to the system of two $\mathrm{K}^{+}$ ions in water.

The total effective force between $\mathrm{K}^{+}$and $\mathrm{Cl}^{-}$from simulation is found to have oscillatory non-monotonic nature, and it is more attractive for a certain range of separation distance typically below $10 \AA$ [Fig. 5(a)]. The distance-dependence of the screened force is modified. Interestingly, the distance dependence of the dielectric function, $\varepsilon_{s}(\mathrm{R})$, extracted from the effective force [Fig. 5(b)] exhibit a wave-like variation with a magnitude substantially lower than 60 at separation distance less than $15 \AA$ A. Similarly, the effective force between two positive charges is more repulsive than the SCFL [Fig. 5(c)] and the extracted $\varepsilon_{s}(\mathrm{R})$ remains much reduced up to $15 \AA$ separation of two $\mathrm{K}^{+}$ions [Fig. 5(d)].

\section{Potential mean force (PMF) between two ions in water}

We computed the potential of mean force $[\mathrm{w}(\mathrm{r})]$ between two ions in water that is defined as

$$
\mathrm{w}(\mathrm{r})=-k_{B} \mathrm{~T} \ln \mathrm{g}(\mathrm{r}) .
$$

We used a radial distribution function between two ion pairs $\left(\mathrm{K}^{+}-\mathrm{K}^{+}\right.$and $\left.\mathrm{K}^{+}-\mathrm{Cl}^{-}\right)$in a system of $1 \mathrm{M}$ aqueous $\mathrm{KCl}$ salt solution and obtained the PMF between them. The effective force here includes the contributions from other ionic species and modified polarization force due to their presence. This distance-dependent 

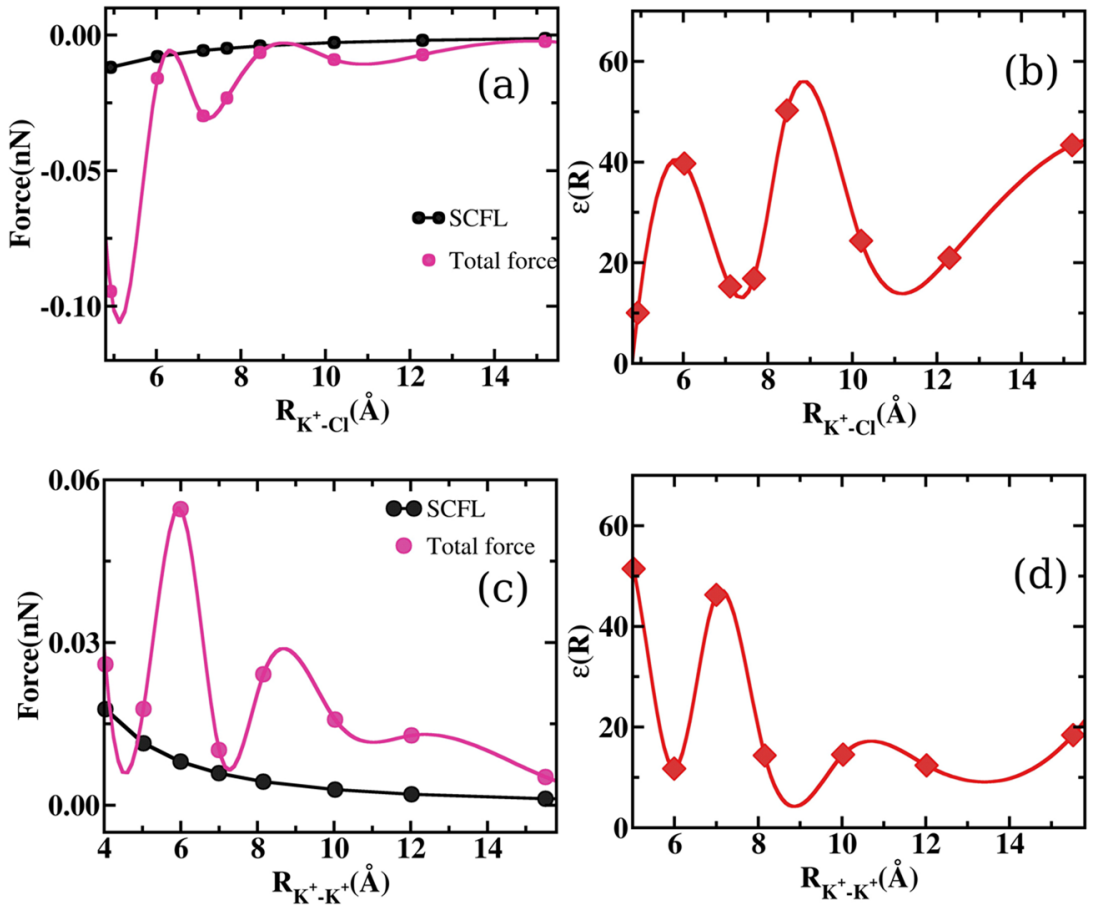

FIG. 5. (a) Comparison of the effective force between two ions $\left(\mathrm{K}^{+}\right.$and $\mathrm{Cl}^{-}$) calculated from simulation with that predicted by the screened Coulomb's force law (SCFL). (b) Distance dependent dielectric function, $\varepsilon(R)$, between ion pairs. Note that usually $r$ is used to define distance dependent dielectric constant everywhere in the literature. We deliberately changed the notation as here the distance between two ions is denoted by $R$; [(c) and (d)] correspond to the system of two $\mathrm{K}^{+}$ions in water.

interaction potential is a mean-field result which differs significantly from the effective force(s) between two charges we have calculated from our simulation (shown in Fig. 5).

Figures 6 deserves to be compared with Figs. 4 and 5. Although there are certain degrees of similarity between them, they are not the same. There are differences in positions of minima and maxima, as well as depths, heights, and the length scale. Note that we should not expect a close agreement because the potential of mean force captures an approximate description including the effects from all the ions present in the system.

\section{E. Correlated ion pair motions in water}

To investigate the effect of polarization induced force on the dynamics of the ion pair, we computed the force-force autocorrelation function, $\mathrm{C}_{\mathrm{FF}}(\mathrm{t})$, for the ion pair $\left(\mathrm{K}^{+}\right.$and $\left.\mathrm{Cl}^{-}\right)$constrained at different separation distances,

$$
C_{F F}(t)=\langle\delta F(0) \cdot \delta F(t)\rangle .
$$

Here, $\delta \mathrm{F}$ is the fluctuation of effective force between two ions along the inter-ionic axis, $k_{B}$ is the Boltzmann constant, $\mathrm{T}$ is the temperature, and the triangular bracket $\langle\cdots\rangle$ denotes the ensemble average. We show the simulated force autocorrelation functions, $\mathrm{C}_{\mathrm{FF}}(\mathrm{t})$, for different inter-ionic distances in Fig. 7. Unlike a free particle, these autocorrelation functions do not show an oscillatory behavior with negative values, and for different separation distances, the decays of correlation functions are not same. For lesser separation distances $\left(\mathrm{R}_{\mathrm{K}^{+}-} \mathrm{Cl}^{-}=3.9 \AA\right.$ $)$ where there are no water molecules present between two ions, the autocorrelation function gets modified further with a much faster decay because the slow contribution from the intervening water is absent.
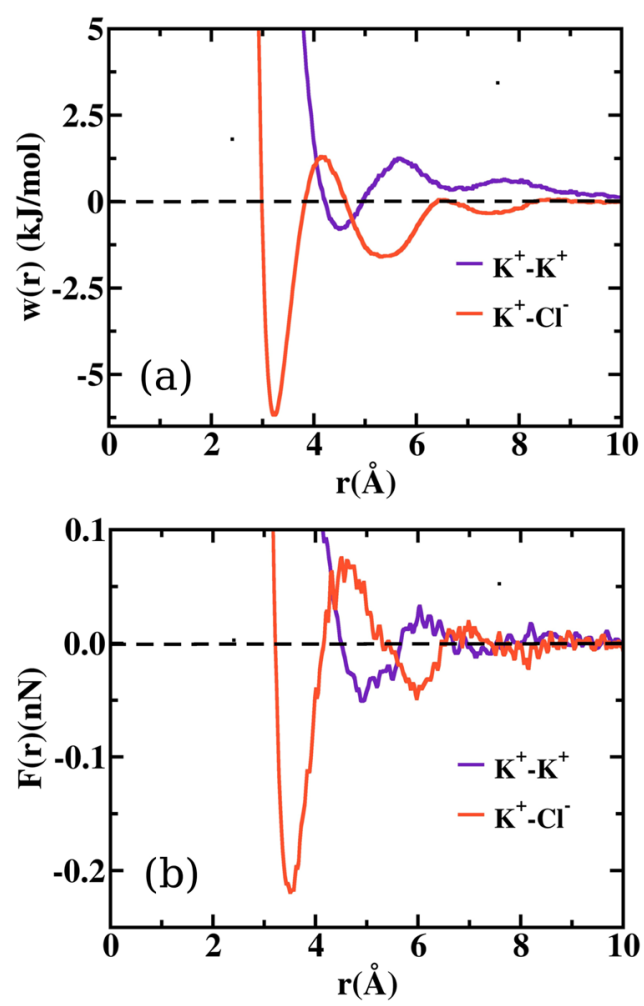

FIG. 6. (a) Potential mean force (PMF) for different ion pairs obtained from the simulation of $1 \mathrm{M}$ aqueous $\mathrm{KCl}$ salt solution. (b) Effective force between ion pairs derived from PMF. 


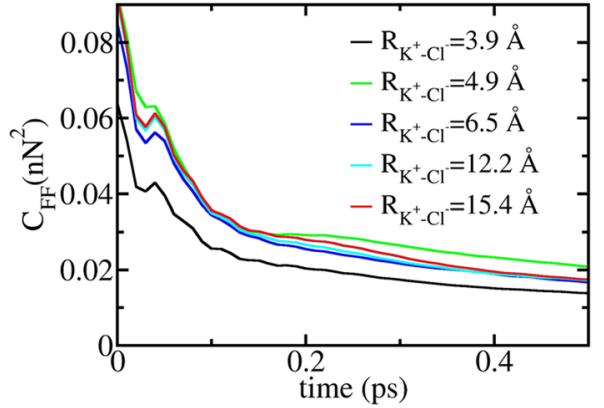

FIG. 7. Force-force time autocorrelation functions with the fluctuation in effective force between $\mathrm{K}^{+}$and $\mathrm{Cl}^{-}$ions, constrained at different separation distances,

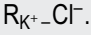

We can write a Langevin equation for this system as

$$
\mu \dot{v}_{12}=F(r)-\zeta_{12}(r) v_{12}+R(r, t)
$$

Here, $\mu$ is the effective mass of the ion pair, $v_{12}$ is their mutual velocity, $\zeta_{12}(r)$ is the distance-dependent friction constant for mutual diffusion, and $\mathrm{R}(\mathrm{r}, \mathrm{t})$ is the random noise term. In the present case, there is a non-zero effective force $[\mathrm{F}(\mathrm{r})]$ between the two ions that arises from the competition of the bare Coulomb's force and polarization force, as discussed earlier. Due to this, a microscopic derivation of the friction coefficient for the mutual diffusion of the ion pair is non-trivial.

One can determine the components of the dielectric friction tensor of the ion pair, constrained at different separation distances through Kirkwood's fluctuation expression,

$$
\zeta_{i j}=\frac{1}{k_{B} T} \int_{0}^{t}\left\langle\delta F_{i}(0) \cdot \delta F_{j}(t)\right\rangle d t .
$$

Here, $\delta F_{i}\left(=F_{i}-\left\langle F_{i}\right\rangle\right)$ is the fluctuation of the effective force on each ion, $k_{\mathrm{B}}$ is the Boltzmann constant, $\mathrm{T}$ is the temperature, and triangular brackets $\langle\cdots\rangle$ denote the ensemble average.

We computed the force on $\mathrm{K}^{+}\left(\mathrm{F}_{1}\right)$ and force on $\mathrm{Cl}^{-}\left(\mathrm{F}_{2}\right)$. From the self force-force correlation function, $\zeta_{11}(\mathrm{t})$ and $\zeta_{22}(\mathrm{t})$ are obtained, and from the cross force-force correlation between two ions, we obtain $\zeta_{12}(\mathrm{t})$. The most interesting result to note here is at shorter separation distances [Figs. 8(a) and $8(\mathrm{~b})$ ], $\zeta_{11}(\mathrm{t})$ and $\zeta_{22}(\mathrm{t})$ are significantly different, but at larger separation distances [Figs. 8(c) and $8(d)$ ], they become similar. This result suggests that the fluctuation of force depends very weakly on the different solvation environment of two different ions as at larger separation, $\zeta_{11}(\mathrm{t})$ and $\zeta_{22}(\mathrm{t})$ are similar. However, at shorter separation distances, the interaction of polarization of two ions results in the different values of $\zeta_{11}(t)$ and $\zeta_{22}(\mathrm{t})$. The relative friction between two ions, $\zeta_{12}(\mathrm{t})$, is always larger than $\zeta_{11}(\mathrm{t})$ and $\zeta_{22}(\mathrm{t})$, and for the separation distance $4.9 \AA$, $\zeta_{12}(t)$ exhibits the highest values compared to other separation distances. However, a projection-operator technique can provide, in principle, an exact friction tensor between two ions for their mutual diffusion.

\section{F. Density and mean-square displacement of water between two ions}

We computed the density profile of water molecules between two oppositely charged ions by separating the inter-ionic region into grids. At a separation of $\sim 5 \AA$, there is only one layer of water molecules although the number density is greater than the bulk
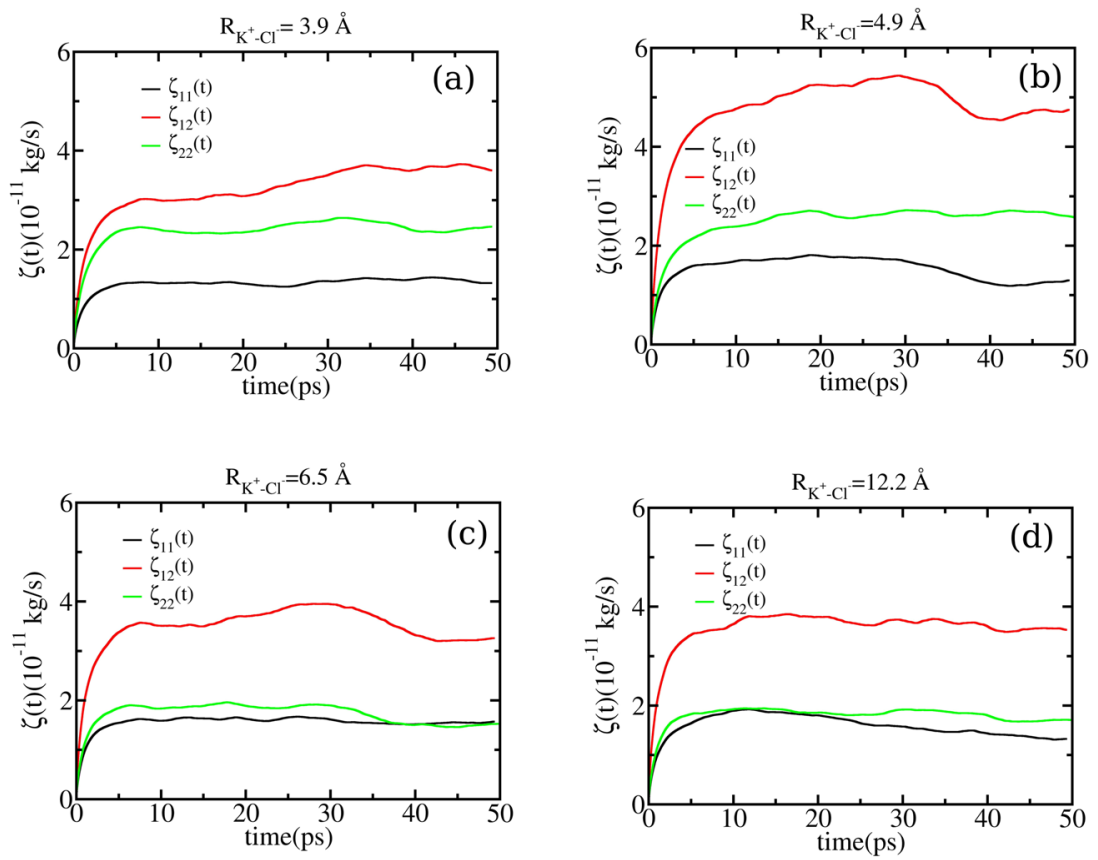

FIG. 8. Components of dielectric friction tensor, arising from self $\left(\zeta_{11}, \zeta_{22}\right)$ and cross $\left(\zeta_{12}\right)$ force-force autocorrelation function. [(a)-(d)] correspond to the simulations of $\mathrm{K}^{+}$and $\mathrm{Cl}^{-}$ion pair fixed at different separation distances. 

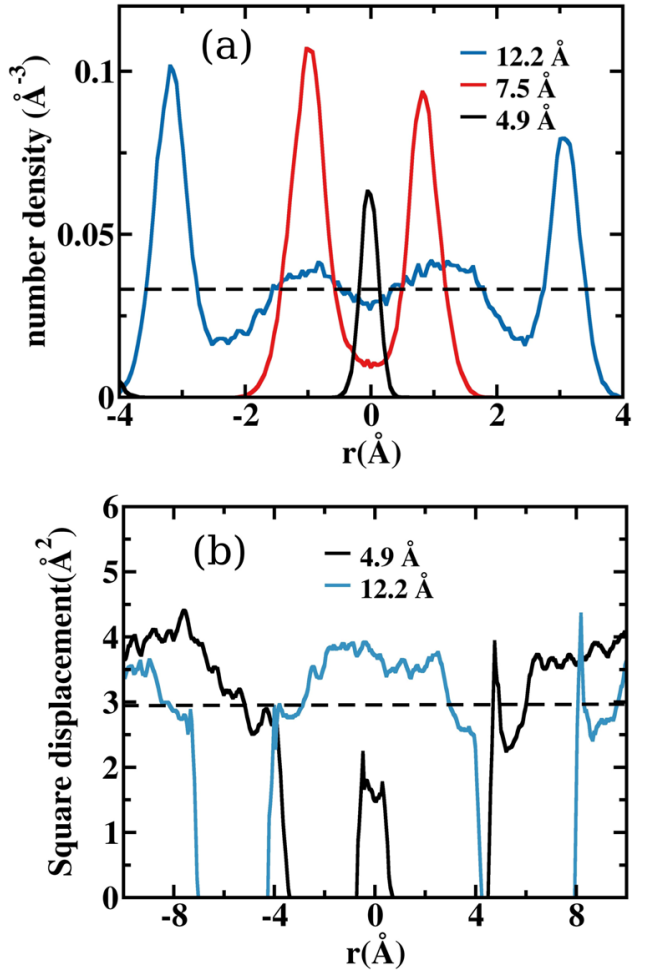

FIG. 9. (a) Density profile of water molecules between $\mathrm{K}^{+}$and $\mathrm{Cl}^{-}$ion pairs. The bulk number density of water is shown by dotted lines. (b) Square displacement of water around the ion pair for the time interval $2 \mathrm{ps}$. The dotted line denotes the square displacement of bulk water in 2 ps.

density [shown by the dotted lines in Fig. 9(a)]. With the increase in the distance of separation, the profile also changes, and after $\sim 10 \AA$, the density profile in the central region of interionic axis becomes similar to the bulk density. The asymmetry found in the two peaks near the ions is real. The density profile reflects the formation of structures in the water molecules between the two ions.

We analyzed the dynamics of those water molecules between $\mathrm{K}^{+}$and $\mathrm{Cl}^{-}$ions. We divided the inter-ionic region into grids and calculated the square displacement (for the time interval $2 \mathrm{ps}$ ) of those water molecules, which were initially (time $t=0$ ) found in the grids. As the polarization of water molecules between two oppositely charged ions increases and the water molecules become more structured, the diffusivity of water molecules also decreases significantly [Fig. 9(b)]. These differences are expected to play a role in determining the rates of various chemical processes.

\section{CONCLUSIONS}

The dielectric screening effect embodied in the PoissonBoltzmann (or generalized Born) description is used widely in simulations of electrolyte solutions and biomolecules. Such treatments assume that each ion experiences an average influence of its surrounding environment. In this description, no ion-ion correlation or fluctuation effect is included that could play an important role in high ion charge density. Similarly, in many theoretical approaches, including Debye-Huckel theory, a primitive model of electrolyte solutions and mean spherical approximation models also invoke dielectric screening of inter-ionic interactions. Therefore, the ability of the SCFL to predict the interaction between charges determines the success of such theoretical or computational techniques. However, the development of theoretical formulations to study correlations in ionic solutions with the explicit treatment of solvent molecules has a long history starting from late 1970s. In an interesting study using mean-spherical approximation, Chan and coworkers investigated the behavior of solvent molecules around an ion using the model of a hard sphere ion in hard sphere dipoles. ${ }^{39}$

Simulations of an ion in water using continuum models neglect the ion-water as well as water-water interactions, thus treating weakly hydrated and strongly hydrated ions similarly. Although in dilute electrolyte solutions, it works reasonably well, for concentrated electrolyte solutions, continuum electrostatics fails to reproduce experimental results. Besides this, in complex biological environments, such as ion channels, it predicts a significant reduction in polarization of water as the distance from the ion increases. However, atomistic MD simulations show a long-range correlation of water dipoles with the distance from the ion in the channel.

Any microscopic theoretical formalism must obey screened Coulomb's force law at larger ion-ion separation distance. A microscopic expression for polarization density, $\mathrm{P}(\mathrm{r})$, in a dipolar liquid is given by

$$
\mathrm{P}(\mathrm{r})=\mu \int \mathrm{d} \boldsymbol{\omega} \hat{\alpha}(\boldsymbol{\omega}) \rho(\mathbf{r}, \boldsymbol{\omega}),
$$

where $\mu$ is the magnitude of the dipole moment of a solvent molecule, $\rho(\mathbf{r}, \boldsymbol{\omega})$ is the position and orientation dependent solvent density, and $\hat{\alpha}(\boldsymbol{\omega})$ is a unit vector with orientation $\boldsymbol{\omega}$. As discussed elsewhere, ${ }^{40}$ one can use classical density functional theory (DFT) to obtain a free energy in terms of the polarization field, ${ }^{33,40}$

$$
\begin{aligned}
\beta F[\mathbf{P}(\mathbf{k})] \cong & \frac{1}{3 \mathrm{y}(2 \pi)^{2}}\left\{\int \mathrm{dkP}^{2}(\mathbf{k})-\frac{\rho_{0}}{3} \int \mathrm{d} \mathbf{k P}(\mathbf{k}) \cdot \mathrm{C}(\mathbf{k}) \cdot \mathrm{P}(-\mathbf{k})\right\} \\
& -(2 \pi)^{-3} \int \mathrm{d} \mathbf{k P}(\mathbf{k}) \cdot \mathrm{E}_{0}(\mathbf{k}),
\end{aligned}
$$

where $\mathrm{E}_{0}(\mathrm{k})$ is the bare electric field of the charged particle, $\mathrm{C}(\mathrm{k})$ is related to the wave number dependent static dielectric tensor of the liquid, and $3 y=4 \pi \beta \rho_{0} \mu^{2} / 3$. $^{5}$ This is the microscopic counterpart of Eq. (5) and it reduces to Eq. (8) under appropriate conditions. In this case, the free energy functional needs to be minimized with respect to $\mathbf{P}(\mathbf{k})$ in the presence of the field due to two ions separated by a distance $R$. This feature makes the evolution of $\mathbf{P}(\mathbf{k})$ [or $\mathbf{P}(\mathbf{r})$ ] highly non-trivial. We hope to return to this problem in future.

The present computer simulation based study reveals several interesting aspects of the effective force between two ions in water. We observe an interesting anti-vertex pair such as the formation of water dipoles around the two positive ions (shown in Fig. 2). The effective force between two ions differs largely from the SCFL for the separation distance less than 10-15.

In summary, we have shown that the interference between the polarizations of water owing to the presence of two ions decides the polarization induced force that adds to the bare Coulomb's law to determine the effective force. The resultant force is found to be 
vastly different from the screened Coulomb's force law at a separation distance less than 10-15 ̊. Therefore, this study partly explains the failure of theories such as Debye-Huckel-Onsager at high concentration and questions the simulation of biomolecules using the Poisson-Boltzmann (or generalized Born) equation for systems with higher charge density. The computed distance dependent dielectric fraction, $\varepsilon_{s}(R)$, reveals that the screening could significantly reduce at intermediate separations, with some multiple minima, which leads to the formation of a contact ion pair, a solvent separated ion pair, etc.

\section{SUPPLEMENTARY MATERIAL}

See the supplementary material for the average dipole moment vector around $\mathrm{K}^{+}-\mathrm{Cl}^{-}$pair and the difference map compared to the single ion situations.

\section{ACKNOWLEDGMENTS}

The authors thank the Department of Science and Technology (DST, India), the Council of Scientific and Industrial Research (CSIR, India) (to P.B.), and Sir J. C. Bose Fellowship (to Professor B. Bagchi) for providing partial financial support.

\section{REFERENCES}

${ }^{1}$ R. A. Marcus, Annu. Rev. Phys. Chem. 15, 155 (1964).

${ }^{2}$ M. Tachiya, J. Phys. Chem. 97, 5911 (1993).

${ }^{3}$ P. J. W. Debye, Polar Molecules (The Chemical Catalog Company, Inc., New York, 1929).

${ }^{4}$ H. Fröhlich, Theory of Dielectrics: Dielectric Constant and Dielectric Loss, Monographs on the physics and chemistry of materials (Clarendon Press, 1958).

${ }^{5}$ B. Bagchi, Molecular Relaxation in Liquids (OUP, USA, 2012).

${ }^{6}$ P. G. Wolynes, Annu. Rev. Phys. Chem. 31, 345 (1980).

${ }^{7}$ R. L. Hayes et al., J. Am. Chem. Soc. 134, 12043 (2012).
${ }^{8}$ T. Yagasaki, S. Saito, and I. Ohmine, J. Phys. Chem. A 114, 12573 (2010).

${ }^{9}$ R. M. Lynden-Bell, Electrochem. Commun. 9, 1857 (2007).

${ }^{10}$ R. M. Lynden-Bell, Phys. Chem. Chem. Phys. 12, 1733 (2010).

${ }^{11}$ M. Tachiya, J. Phys. Chem. 93, 7050 (1989).

${ }^{12}$ A. Chandra and B. Bagchi, J. Phys. Chem. 93, 6996 (1989).

${ }^{13}$ W. R. Fawcett and Y. I. Kharkats, J. Electroanal. Chem. Interfacial Electrochem. 47, 413 (1973).

${ }^{14}$ J. M. Blaney et al., J. Am. Chem. Soc. 104, 6424 (1982).

${ }^{15}$ P. Cieplak et al., Biopolymers 29, 717 (1990).

${ }^{16}$ A. Warshel, S. T. Russell, and A. K. Churg, Proc. Natl. Acad. Sci. U. S. A. 81, 4785 (1984).

${ }^{17}$ A. R. Srinivasan and W. K. Olson, Fed. Proc. 39, 2199 (1980).

${ }^{18}$ B. E. Hingerty et al., Biopolymers 24, 427 (1985).

${ }^{19}$ J. Ramstein and R. Lavery, Proc. Natl. Acad. Sci. U. S. A. 85, 7231 (1988).

${ }^{20}$ C. Schaaf and S. Gekle, Phys. Rev. E 92, 032718 (2015).

${ }^{21}$ D. Borgis, L. Belloni, and M. Levesque, J. Phys. Chem. Lett. 9, 3698 (2018).

${ }^{22}$ L. Belloni, D. Borgis, and M. Levesque, J. Phys. Chem. Lett. 9, 1985 (2018).

${ }^{23}$ D. M. Wilkins et al., J. Chem. Phys. 146, 181103 (2017).

${ }^{24}$ J. Duboisset and P.-F. Brevet, Phys. Rev. Lett. 120, 263001 (2018).

${ }^{25}$ J. Høye and G. Stell, J. Chem. Phys. 68, 4145 (1978).

${ }^{26}$ J. Høye and G. Stell, J. Chem. Phys. 71, 1985 (1979).

${ }^{27}$ Y. X. Chen et al., Sci. Adv. 2, e1501891 (2016).

${ }^{28}$ E. Pluhařová, D. Laage, and P. Jungwirth, J. Phys. Chem. Lett. 8, 2031 (2017).

${ }^{29}$ R. Biswas et al., J. Chem. Phys. 137, 014515 (2012).

${ }^{30}$ A. Chandra and B. Bagchi, J. Chem. Phys. 113, 3226 (2000).

${ }^{31}$ J. F. Dufreche et al., Phys. Rev. Lett. 88, 095902 (2002).

${ }^{32}$ J. F. Dufreche et al., J. Phys. Chem. B 112, 10264 (2008).

${ }^{33}$ A. Chandra and B. Bagchi, J. Chem. Phys. 90, 1832 (1989).

${ }^{34}$ S. Plimpton, J. Comput. Phys. 117, 1 (1995).

${ }^{35}$ H. J. C. Berendsen, J. R. Grigera, and T. P. Straatsma, J. Phys. Chem. 91, 6269 (1987).

${ }^{36}$ S. Koneshan et al., J. Phys. Chem. B 102, 4193 (1998).

${ }^{37}$ T. Darden, D. York, and L. Pedersen, J. Chem. Phys. 98, 10089 (1993).

${ }^{38}$ U. Essmann et al., J. Chem. Phys. 103, 8577 (1995).

${ }^{39}$ D. Y. Chan, D. J. Mitchell, and B. W. Ninham, J. Chem. Phys. 70, 2946 (1979).

${ }^{40}$ A. Chandra and B. Bagchi, J. Chem. Phys. 94, 2258 (1991). 